\title{
X-ray Observations of High-B Radio Pulsars
}

\author{
S. A. Olausen ${ }^{1}$, W. W. Zhu ${ }^{2}$, J. K. Vogel ${ }^{3}$, V. M. Kaspi ${ }^{1}$, A. G. Lyne ${ }^{4}$, C. M. Espinoza ${ }^{4}$, B. W. \\ Stappers $^{4}$, R. N. Manchester ${ }^{5}$, \& M. A. McLaughlin 6
}

\begin{abstract}
The study of high-magnetic-field pulsars is important for examining the relationships between radio pulsars, magnetars, and X-ray-isolated neutron stars (XINSs). Here we report on X-ray observations of three such high-magnetic-field radio pulsars. We first present the results of a deep XMM-Newton observation of PSR J1734-3333, taken to follow up on its initial detection in 2009. The pulsar's spectrum is well fit by a blackbody with a temperature of $300 \pm 60 \mathrm{eV}$, with bolometric luminosity $L_{\mathrm{bb}}=2.0_{-0.7}^{+2.2} \times$ $10^{32} \mathrm{erg} \mathrm{s}^{-1} \approx 0.0036 \dot{E}$ for a distance of $6.1 \mathrm{kpc}$. We detect no X-ray pulsations from the source, setting a $1 \sigma$ upper limit on the pulsed fraction of $60 \%$ in the $0.5-3 \mathrm{keV}$ band. We compare PSR J1734-3333 to other rotation-powered pulsars of similar age and find that it is significantly hotter, supporting the hypothesis that the magnetic field affects the observed thermal properties of pulsars. We also report on XMM-Newton and Chandra observations of PSRs B1845-19 and J1001-5939. We do not detect either pulsar, setting $3 \sigma$ upper limits on their blackbody temperatures of 48 and $56 \mathrm{eV}$, respectively. Despite the similarities in rotational properties, these sources are significantly cooler than all but one of the XINSs, which we attribute to the two groups having been born with different magnetic fields and hence evolving differently.
\end{abstract}

Subject headings: pulsars: general — pulsars: individual (PSR B1845-19, PSR J1001-5939, PSR J1734-3333) — stars: neutron — X-rays: stars

\footnotetext{
${ }^{1}$ Department of Physics, Rutherford Physics Building, McGill University, 3600 University Street, Montreal, Quebec, H3A 2T8, Canada

${ }^{2}$ Department of Physics and Astronomy, University of British Columbia, 6224 Agricultural Road, Vancouver, BC, V6T 1Z1, Canada

${ }^{3}$ Lawrence Livermore National Laboratory, 7000 East Avenue, Livermore, CA 94550, USA

${ }^{4}$ Jodrell Bank Centre for Astrophysics, School of Physics and Astronomy, University of Manchester, Manchester, M13 9PL, UK

${ }^{5}$ Australia Telescope National Facility, CSIRO Astronomy and Space Science, Epping, NSW 1710, Australia

${ }^{6}$ Department of Physics, West Virginia University, White Hall, Morgantown, WV 26506, USA
} 


\section{Introduction}

Over the past few decades, the boom in X-ray and gamma-ray astronomy has led to a significant increase in our knowledge about the neutron star family. Previously, the only kind of isolated neutron stars known were radio pulsars. The latter are also known as 'rotation-powered pulsars' (RPPs) because their luminosities are generally much lower than $\dot{E}$, the rate of energy loss due to rotation. Since then, X-ray observations have led to the discovery of several new classes of isolated neutron stars, including magnetars and X-ray-isolated neutron stars (XINS\$1 1); see Kaspi (2010) for a review. All of these classes exhibit properties different from those of conventional RPPs.

Magnetars, including the soft gamma repeaters (SGRs) and anomalous X-ray pulsars (AXPs), are isolated, slowly rotating neutron stars. The known object:2 have spin periods in the range of 2-12s, X-ray luminosities that are in many cases much higher than their spin-down luminosities, and, assuming conventional magnetic dipole braking, typically very high magnetic fields $\left(B \sim 10^{14}-10^{15} \mathrm{G}\right)$. It is generally believed that their X-ray luminosities are fueled by the decay of these high magnetic fields (Thompson \& Duncan 1995, 1996; Thompson et al. 2002). Magnetars also show great X-ray variability and activity, ranging from short SGR-like X-ray $/ \gamma$-ray bursts to major, slow-rise and long-lived X-ray flares, that are thought to be produced by fracturing of the crust and reconfiguration of the magnetic field lines. For reviews of magnetars, see for example Woods \& Thompson (2006) or Mereghetti (2008).

The XINSs (see Haberl 2007 and Turolla 2009 for reviews) are a group of nearby (distance $\leq 500 \mathrm{pc}$ ) neutron stars characterized by soft thermal X-ray spectra and no detected radio emission. Those XINSs with detailed timing measurements have spin periods in the range of $3-11 \mathrm{~s}$, high inferred magnetic fields $\left(B \sim(1-3) \times 10^{13} \mathrm{G}\right)$, and characteristic ages of $\sim 10^{6}$ yr (Kaplan \& van Kerkwijk 2009, 2011; van Kerkwijk \& Kaplan 2008). The XINSs are considerably hotter and more X-ray luminous than similarly aged ordinary RPPs (Kaplan \& van Kerkwijk 2009), although they show no bursting activity and are less luminous than the magnetars. Therefore, it has been proposed (Kaplan \& van Kerkwijk 2009; Pons et al. 2009; Popov et al. 2010) that the cooling of XINSs is affected by heating of the neutron-star surface due to magnetic-field decay. If correct, this suggests that XINSs may be evolutionarily related to the magnetars; one possibility is that XINSs are magnetar descendants, being older versions of neutron stars that were born with $10^{14}-10^{15} \mathrm{G}$ magnetic fields which have slowly decayed with time.

The hint of magnetic field decay heating in XINSs suggests that similar processes may be occuring in radio pulsars that have ages and magnetic fields comparable to those of the INSs. Thanks to radio surveys over the past decade (e.g. Manchester et al. 2001; Morris et al. 2002), a class of radio pulsar having inferred magnetic fields comparable to XINSs and even bona fide magnetars has emerged. What physically differentiates these high- $B$ RPPs from these other classes

\footnotetext{
${ }^{1}$ Also known as X-ray dim isolated neutron stars (XDINSs).

${ }^{2}$ See the McGill SGR/AXP Online Catalog at http://www.physics.mcgill.ca/ pulsar/magnetar/main.html.
} 
is unclear; aside from their radio emission, which was selected for via their discovery in radio surveys, their X-ray emission properties may well be similar to those of the XINSs and magnetars. Indeed, in 2009 the High Time Resolution Universe survey discovered a radio pulsar whose rotational and X-ray properties identified it as a magnetar (Levin et al. 2010).

Here we present X-ray observations of three high- $B$ radio pulsars: PSRs J1734-3333, B1845-19, and J1001-5939. These as a group are key sources for X-ray study, as they share spin, and hence spin-inferred properties with the XINSs and magnetars. Thus they might be expected to share $\mathrm{X}$-ray properties, too.

PSR J1734-3333 has period $P=1.17 \mathrm{~s}$ and period derivative $\dot{P}=2.3 \times 10^{-12}$ and was discovered in the Parkes Multibeam Survey (Morris et al. 2002). Its spin parameters imply a spindown luminosity of $\dot{E} \equiv 4 \pi^{2} I \dot{P} / P^{3}=5.6 \times 10^{34} \mathrm{erg} \mathrm{s}^{-1}$, characteristic age of $\tau \equiv P / 2 \dot{P}=8.1 \mathrm{kyr}$, and an inferred surface dipolar magnetic field of $B \equiv 3.2 \times 10^{19}(P \dot{P})^{1 / 2} \mathrm{G}=5.2 \times 10^{13} \mathrm{G}$, which is among the highest of all known radio pulsars and similar to those of bona fide magnetars such as 1E $2259+586\left(B=5.9 \times 10^{13} \mathrm{G}\right.$; Kaspi et al. 1999$)$ and Swift J1822.3-1606 $\left(B=4.7 \times 10^{13} \mathrm{G}\right.$; Livingstone et al. 2011; Scholz et al. 2012; but see Rea et al. 2012). It has a radio dispersion measure (DM) of $578 \mathrm{pc} \mathrm{cm}^{-3}$ which, based on the NE2001 model for Galactic free electron density (Cordes \& Lazio 2002), gives a best-estimated distance to the pulsar of $6.1 \mathrm{kpc}$ (although these distance estimates typically have large uncertainties of $25 \%$ or more). Based on its unusually low braking index $(n=0.9 \pm 0.2)$, Espinoza et al. (2011) suggested that the pulsar's magnetic field is growing, i.e. its trajectory on a conventional $P / \dot{P}$ diagram is up and to the right, towards the region occupied by the magnetars. To search for anomalous X-ray emission from the pulsar, a short XMM-Newton observation was taken in 2009. The source was detected, and although there were indications that it was anomalously hot, it was too faint and the observation too short to determine the spectrum unambiguously or detect pulsations (Olausen et al. 2010).

PSR B1845-19 is a 4.31-s period radio pulsar discovered in the Molonglo pulsar survey (Manchester et al. 1978). It has $\dot{P}=2.33 \times 10^{-14}, \dot{E}=1.1 \times 10^{31} \mathrm{erg} \mathrm{s}^{-1}, \tau=2.9 \mathrm{Myr}$, and $B=1.0 \times 10^{13} \mathrm{G}$. It has a DM of $18 \mathrm{pc} \mathrm{cm}^{-3}$, implying a distance of $0.75 \mathrm{kpc}$ (Cordes \& Lazio 2002). The pulsar's magnetic field, age, $\dot{E}$ and distance are close to those of the XINSs.

PSR J1001-5939 was another pulsar discovered in the Parkes Multibeam Pulsar Survey (Lorimer et al. 2006), with period $P=7.73 \mathrm{~s}$ and period derivative $\dot{P}=5.99 \times 10^{-14}$. As with PSR B1845-19, the low $\dot{E}\left(5.1 \times 10^{30} \mathrm{erg} \mathrm{s}^{-1}\right)$, age $(\tau=2.1 \mathrm{Myr})$, and high magnetic field $\left(B=2.2 \times 10^{13} \mathrm{G}\right)$ of PSR J1001-5939 are similar to the properties of the known XINSs, although at a distance of $2.7 \mathrm{kpc}$ (as determined from its DM of $113 \mathrm{pc} \mathrm{cm}^{-3}$ ) it is farther away than any of them.

In this paper, we report on X-ray observations of these three high- $B$ RPPs, done with the aim of searching for magnetar- or XINS-like emission as might be expected if magnetic-field-decay heating is occuring. Section 2 describes our X-ray observations, Section 3 our results, and we discuss the implications of our findings in Section 4 . 


\section{Observations}

\subsection{PSR J1734-3333}

A deep, 125-ks observation of PSR J1734-3333 was carried out on 2011 March 11-12 with the $X M M-N e w t o n$ observatory (Jansen et al. 2001). We also reanalyzed the EPIC pn data from the 10-ks 2009 XMM-Newton observation of the source (Olausen et al. 2010), but not the MOS data, as they had neither the proper time resolution nor sufficient source counts to meaningfully contribute to our analysis. In both observations, the EPIC pn camera (Strüder et al. 2001) was operating in large-window mode and the EPIC MOS cameras (Turner et al. 2001) in full-window mode; the medium filter was in use for all three cameras. Details of the two observations, as well as those described in Sections 2.2 and 2.3, are summarized in Table 11. The data from both observations were analyzed with the XMM Science Analysis System (SAS) version 11.0.03. To search for times of high background flaring that are known to sometimes affect XMM-Newton data, we extracted light curves from over the entire field of view of all three cameras. The 2011 observation was heavily affected, with over half the exposure length contaminated by background flares. The 2009 observation showed no such problems.

\subsection{PSR B1845-19}

PSR B1845-19 was observed for $38 \mathrm{ks}$ by XMM-Newton on 2011 March 16. The pn camera was operating in large-window mode with the thin filter, and the MOS cameras were operating in full-window mode also using the thin filter (see Table 1 for further details). The data were reduced with XMM SAS 11.0.0, and times of high background flaring were removed. Because the two MOS cameras are similar instruments, we combined their images for the analysis of these data.

\subsection{PSR J1001-5939}

On 2011 October 8, the Chandra X-ray Observatory observed the position of PSR J1001-5939 (see Table 1) for $19 \mathrm{ks}$, using the back-illuminated S3 chip of the Advanced CCD Imaging Spectrometer (ACIS; Garmire et al. 2003) as aimpoint. The 1/8 subarray mode was chosen to achieve a time resolution of $0.44 \mathrm{~s}$, sufficient for timing a pulsar of period $7.73 \mathrm{~s}$. For our analysis, we worked with the level 2 event files, which were created from level 1 event lists by including good time intervals as well as information about issues such as bad pixels and cosmic rays. The data were analyzed using the tools provided in CIAO 4.3 .

\footnotetext{
${ }^{3}$ See http://xmm.esac.esa.int/sas/

${ }^{4}$ See http://cxc.harvard.edu/ciao/
} 


\section{Results}

\subsection{PSR J1734-3333}

\subsubsection{Imaging}

Using the SAS tool edetect_chain to perform a blind search for point sources, we detected the X-ray counterpart of PSR J1734-3333 in both the 2009 and 2011 observations, with the reported count rates in both observations being consistent with each other. Our best-fit position for the X-ray source is the one reported by edetect_chain for the 2011 observation: (J2000) R.A. $=17^{\mathrm{h}} 34^{\mathrm{m}} 27^{\mathrm{s}} .03(4)$, decl. $=-33^{\circ} 33^{\prime} 22^{\prime \prime} \cdot 4(5)$, where the listed uncertainties are the statistical errors only. It is consistent with the radio timing position of R.A. $=17^{\mathrm{h}} 34^{\mathrm{m}} 26.9(2)$, decl. $=$ $-33^{\circ} 33^{\prime} 20^{\prime \prime}(10)$ (Espinoza et al. 2011). However, Olausen et al. (2010) also identified an optical source (NOMAD Catalog5 ID 0564-0621454) that lay within the 2009 X-ray error circle and determined that either it or the radio pulsar could be associated with the X-ray source, but not both. We find that the updated X-ray position excludes the optical source, even when including XMM-Newton's 2" absolute pointing uncertainty, strengthening the conclusion from Olausen et al. (2010) that the X-ray source is the counterpart to PSR J1734-3333. We also found that the radial profile of the X-ray source is consistent with the $X M M$ point-spread function (PSF). Therefore there is no evidence of extended emission.

\subsubsection{Timing Analysis}

To search for X-ray pulsations from PSR J1734-3333, we extracted counts in the $0.5-3 \mathrm{keV}$ energy band from a region of $30^{\prime \prime}$ radius centered on the source. The MOS cameras, operating with a time resolution of $2.6 \mathrm{~s}$, were unsuitable for this analysis, so only the pn data were used. Within the 2011 source region we found 576 counts, and by extracting counts in the same energy range from source-free regions on the same CCDs, we determined that $352 \pm 12$ of those counts were from the background. Following the same procedure for the 2009 data we determined that of the 150 total counts in the source region, $100 \pm 6$ were from background photons. After barycentering the source region events with the SAS tool barycen, we folded them into eight phase bins based on a radio timing ephemeris obtained using the Jodrell Bank 76-m Lovell telescope (Espinoza et al. 2011). Since the time span covered by the radio ephemeris included the epochs of both the 2009 and 2011 observations, we could produce a single summed light curve from both sets of data.

Fitting the folded curve to a straight line gave a best-fit $\chi^{2}$ of 11.1 for 7 degrees of freedom, or a $13 \%$ chance that the folded curve could result with no signal in the data. Therefore, no

\footnotetext{
5 http://www . usno.navy.mil/USNO/astrometry/optical-IR-prod/nomad

${ }^{6}$ See http://xmm2.esac.esa.int/docs/documents/CAL-TN-0018.pdf
} 
significant pulsations were detected. We also performed additional searches, attempting to improve the signal-to-noise ratio by using an energy range of $1-2 \mathrm{keV}$ or a source region of $20^{\prime \prime}$ radius, as well as searching around the predicted period, but in all cases there were again no detections of pulsations.

To find an upper limit for the pulsed fraction, we simulated event lists with the same number of total counts as found in the source region. The simulated signal had a sinusoidal profile with a random phase and had a user-specified area pulsed fraction, where the latter is defined as the ratio of the pulsed part of the profile to the entire profile. We found that a signal with area pulsed fraction of $\sim 0.23$ would be detected with $>3 \sigma$ significance $68 \%$ of the time. Therefore, because $62 \%$ of the counts in the source region are from the background, we estimate that the $1 \sigma$ upper limit on the area pulsed fraction of PSR J1734-3333 is 0.6 in the $0.5-3 \mathrm{keV}$ energy range.

\subsubsection{Spectral Analysis}

We extracted the spectrum of PSR J1734-3333 from the 2011 pn and MOS data using a region of $30^{\prime \prime}$ radius centered on the X-ray source position. Background regions were chosen from nearby, source-free areas on the same CCD as the pulsar, and response and ancillary response files were generated with the SAS tasks rmfgen and arfgen. The pn and MOS spectra were grouped to have a minimum of 25 counts per bin. We also extracted a spectrum from the 2009 pn data using the same source and background regions as described in Olausen et al. (2010), grouped to have a minimum of 20 counts per bin.

These four spectra were then jointly fit in XSPEC 12.7.0 using simple power-law and blackbody models, as well as with nsa (Pavlov et al. 1995) and nsmax (Ho et al. 2008) neutron-star atmosphere models, all using phabs to model interstellar absorption. The absorbed power-law model provided a statistically acceptable fit to the data $\left(\chi^{2}=34.6\right.$ for 44 degrees of freedom), but as it gave an unphysically steep photon index of 5.2, we do not consider it any further. Best-fit parameters for the other three models are shown in Table 2 .

The best-fit blackbody model is plotted in Figure 1. We found a best-fit7 temperature of $k T=$ $0.30 \pm 0.06 \mathrm{keV}$, corresponding to a column density of $N_{\mathrm{H}}=0.67_{-0.24}^{+0.36} \times 10^{22} \mathrm{~cm}^{-2}$. The blackbody radius is $R_{\mathrm{bb}}=0.45_{-0.20}^{+0.55} d_{6.1} \mathrm{~km}$, giving a bolometric luminosity of $L_{\mathrm{bb}}=2.0_{-0.7}^{+2.2} \times 10^{32} d_{6.1}^{2} \mathrm{erg} \mathrm{s}^{-1}$, where $d_{6.1}$ is the distance to the pulsar in units of $6.1 \mathrm{kpc}$. In order to better explore the confidence range of $k T$ and $N_{\mathrm{H}}$ for the blackbody model, we plotted their confidence contours in Figure 2 , The plot shows that the $3 \sigma$ lower limit on $k T$ is $0.18 \mathrm{keV}$ and that lower values of $k T$ require higher values of $N_{\mathrm{H}}$. We note that the maximum column density along the line of sight to the pulsar 8 is $N_{\mathrm{H}}=(1.1-1.4) \times 10^{22} \mathrm{~cm}^{-2}$, and because the DM distance places the pulsar less than halfway

\footnotetext{
${ }^{7}$ All errors in this section are $90 \%$ confidence intervals.
}

${ }^{8}$ http://heasarc.nasa.gov/cgi-bin/Tools/w3nh/w3nh.pl 
through the Galaxy, the expected column density is significantly lower than that. As a result, models with $k T<0.2 \mathrm{keV}$ which require $N_{\mathrm{H}}>1 \times 10^{22} \mathrm{~cm}^{-2}$ are further disfavored.

Both of the neutron-star atmosphere models provide good fits to the data and yield much lower best-fit temperatures than the blackbody model, as is typical. The nsa model gives $k T^{\infty}=$ $0.14 \pm 0.05 \mathrm{keV}$, but because it assumes that the emission is from the entire surface of the star, it implies an unreasonably large distance to the pulsar of $\sim 27 \mathrm{kpc}$. Fixing the distance at $6.1 \mathrm{kpc}$ results in an even lower temperature of $90 \pm 3 \mathrm{eV}\left(\chi^{2}=37.6\right.$ for 45 degrees of freedom), but the required column density rises to $N_{\mathrm{H}}=1.2_{-0.1}^{+0.2} \times 10^{22} \mathrm{~cm}^{-2}$, which is not favored as described above. Given these difficulties, we also fit the spectrum to the nsmax model for a highly magnetized neutron star atmosphere. This model gives $k T^{\infty}=0.13_{-0.04}^{+0.05} \mathrm{keV}, N_{\mathrm{H}}=0.88_{-0.28}^{+0.40} \times 10^{22} \mathrm{~cm}^{-2}$, and an emission radius of $R=3.2_{-2.0}^{+8.9} d_{6.1} \mathrm{~km}$. Finally, we note that the nsa and nsmax models were fit using the highest available values for the magnetic field (in XSPEC), $10^{13}$ and $2 \times 10^{13} \mathrm{G}$, respectively. Since these values are below the $5.2 \times 10^{13} \mathrm{G}$ field of PSR J1734-3333, the results are not completely reliable.

\subsection{PSR B1845-19}

We employed the SAS tool edetect_chain to search for the X-ray counterpart of PSR B1845-19, but detected no X-ray sources within $1.5^{\prime}$ of the pulsar's radio timing position. From the pn image, we found 561 counts in a circular region of radius $28^{\prime \prime}$ centered on the pulsar position and 781 counts from an annular region of outer radius $45^{\prime \prime}$ around the source region. From the combined MOS image, we found 132 counts in a $27^{\prime \prime}$ radius source region and 192 counts in a surrounding annulus with outer radius $45^{\prime \prime}$. In both cases the source regions were chosen such that they encircle $\sim 80 \%$ of the energy from the pulsar according to the on-axis PSFs of the instruments. The background regions are expected to contain a further $\sim 10 \%$ of the source energy. To estimate the count rate upper limit of the pulsar, we input these values into the aprates tool from CIAO 4.4 and obtained a $3 \sigma\left(99.7 \%\right.$ confidence) upper limit of 0.0048 counts s$^{-1}$ for the pn camera and 0.0029 counts s$^{-1}$ for the combined MOS camera. All counts and count rates are for the $0.2-3 \mathrm{keV}$ energy band.

In order to convert the count rate upper limit into an upper limit on the pulsar's surface temperature $k T$, we assume the pulsar's spectrum can be described by an absorbed blackbody model. We use XSPEC to find the spectrum for different values of the model parameters (blackbody radius $R_{\mathrm{bb}}$, distance, and $N_{\mathrm{H}}$ ) and, based on response and ancillary response files generated for the source regions described previously, calculate the predicted count rates for the pn and MOS. We select 5,10 , and $15 \mathrm{~km}$ as our possible values for $R_{\mathrm{bb}}$, and we use the DM distance to the pulsar of $0.75 \mathrm{kpc}$. The total column density along the pulsar's line of sight ${ }^{8}$ is $N_{\mathrm{H}}=(0.14-0.18) \times 10^{22} \mathrm{~cm}^{-2}$, so we evaluate $k T$ upper limits for a range of column densities with $0 \mathrm{~cm}^{-2}<N_{\mathrm{H}}<0.2 \times 10^{22} \mathrm{~cm}^{-2}$ and calculate bolometric luminosity based on $k T$ and $R_{\mathrm{bb}}$.

We found that the upper limits from the combined MOS cameras provide less strict constraints 
than those of the pn camera, so we present only the upper limits from the pn image of PSR B1845-19 here. These upper limits, in both $k T$ and bolometric luminosity, are shown in Figure 3 , Taking the rather conservative values of $N_{\mathrm{H}}=0.2 \times 10^{22} \mathrm{~cm}^{-2}$ and $R_{\mathrm{bb}}=10 \mathrm{~km}$, our $3 \sigma$ upper limits on $k T$ and bolometric luminosity are $48 \mathrm{eV}$ and $6.8 \times 10^{31} \mathrm{erg} \mathrm{s}^{-1} \approx 6.2 \dot{E}$.

\subsection{PSR J1001-5939}

No X-ray signal was observed at the expected radio position of PSR J1001-5939. We extracted 0 counts over the $0.3-3 \mathrm{keV}$ energy range from a $5^{\prime \prime}$ radius circular region centered on the pulsar position and 141 counts in the same energy range from a surrounding annular region with outer radius $60^{\prime \prime}$. The radius of the source region was chosen such that it contains $\sim 99 \%$ of the expected signal based on the Chandra on-axis PSF; the rest of the potential signal should be present in the background annulus. From these values the CIAO aprates tool gives a $3 \sigma$ upper limit on the pulsar's $0.3-3 \mathrm{keV}$ count rate of $0.34 \times 10^{-3}$ counts $^{-1}$.

Using the same procedure as outlined above for PSR B1845-19, we can obtain upper limits for the surface temperature of PSR J1001-5939 from our count rate upper limits. We again choose blackbody radii of $R_{\mathrm{bb}}=5,10$, and $15 \mathrm{~km}$, and use the DM distance to the pulsar of $2.7 \mathrm{kpc}$. Based on the DM distance, we also estimate the column density to be $N_{\mathrm{H}}=0.33 \times 10^{22} \mathrm{~cm}^{-2}$ from the 3-dimensional Galactic extinction $\left(A_{V}\right)$ model of Drimmel et al. (2003) and the relation $N_{\mathrm{H}} / A_{V}=1.79 \times 10^{21} \mathrm{~cm}^{-2}$ in Predehl \& Schmitt (1995). Therefore, we evaluate $k T$ and luminosity upper limits for $0.1 \times 10^{22} \mathrm{~cm}^{-2}<N_{\mathrm{H}}<0.55 \times 10^{22} \mathrm{~cm}^{-2}$. Note that the total $N_{\mathrm{H}}$ along the line of sight to the pulsar ${ }^{8}$ is $(0.80-0.85) \times 10^{22} \mathrm{~cm}^{-2}$.

Figure 4shows our results for the $k T$ and bolometric luminosity upper limits of PSR J1001-5939. In particular, for our estimate of $N_{\mathrm{H}}=0.33 \times 10^{22} \mathrm{~cm}^{-2}$ and assuming a blackbody radius of $10 \mathrm{~km}$, we find $3 \sigma$ upper limits of $56 \mathrm{eV}$ for $k T$ and $1.26 \times 10^{32} \mathrm{erg} \mathrm{s}^{-1} \approx 25 \dot{E}$ for the bolometric luminosity.

\section{Discussion}

\subsection{PSR J1734-3333}

We have reported on a new long XMM-Newton observation of the young, high- $B$ pulsar PSR J1734-3333. We found a more precise position for the X-ray counterpart that falls within the error ellipse of the pulsar's radio timing position. We were unable to detect X-ray pulsations, and the $1 \sigma$ upper limit of $60 \%(0.5-3 \mathrm{keV})$ that we set on the pulsed fraction is not very constraining.

PSR J1734-3333 has a thermal spectrum, well fit by a blackbody model with an unusually high temperature, $k T=0.30 \pm 0.06 \mathrm{keV}$, and corresponding blackbody radius of $0.45_{-0.20}^{+0.55} d_{6.1} \mathrm{~km}$. The bolometric luminosity is $L_{\mathrm{bb}}=2.0 \times 10^{32} d_{6.1}^{2} \mathrm{erg} \mathrm{s}^{-1}$, giving an X-ray efficiency of $L_{\mathrm{bb}} / \dot{E}=$ 
$0.0036 d_{6.1}^{2}$. The pulsar's small emission radius is suggestive of thermal emission from heated polar caps, but such models predict X-ray efficiencies less than $10^{-3}$. For example, the polar cap reheating model of Harding \& Muslimov (2001) predicts $L_{\mathrm{bb}} / \dot{E} \approx 3 \times 10^{-4}$ for PSR J1734-3333, an order of magnitude below what we observe. Of course, if the DM distance is incorrect and the true distance to the pulsar is within $2 \mathrm{kpc}\left(d_{6.1} \lesssim 0.3\right)$, then the X-ray efficiency drops to roughly what is predicted for polar cap reheating. However, Harding \& Muslimov (2001) warn their model may not apply to pulsars with $B \gtrsim 4 \times 10^{12} \mathrm{G}$ so these predictions are unreliable.

The pulsar's blackbody temperature of $0.30 \mathrm{keV}$ is almost three times as high as expected from a minimal cooling model $(0.07-0.11 \mathrm{keV}$; Page et al. 2006) given its age. Even the neutronstar atmosphere models give best-fit temperatures that are too high, $k T^{\infty}=0.13-0.14 \mathrm{keV}$, though their uncertainties do overlap with the range of predicted cooling temperatures. For instance, the nsa model with distance fixed at $6.1 \mathrm{kpc}$ gives $k T^{\infty}=0.09 \mathrm{keV}$. However, note that as with the simple blackbody, lower temperatures are correlated with higher $N_{\mathrm{H}}$ in the atmosphere models too, which can disfavor that region of parameter space as explained in Section 3.1.3.

Although our ignorance regarding the correct atmosphere model for PSR J1734-3333 precludes meaningful comparisons of its X-ray emission with models of neutron star cooling, we can nevertheless compare our results with those of other RPPs of comparable age. We do this in Figure 5 where we plot blackbody temperature versus characteristic age for RPPs, XINSs, and magnetars. High- $B$ sources are shown in red and yellow (where "high" means $B>10^{13} \mathrm{G}$ ). Although a blackbody model neglects atmospheric effects and hence is almost certainly incorrect for these sources, it provides a simple spectral parameterization by which to describe all source spectra and allows a consistent comparison of spectral properties, even if ultimately not fully physical. Figure 5 shows that PSR J1734-3333 has a much higher blackbody temperature than nearly all other radio pulsars for which this quantity has been measured, with the possible exception of PSR J1119-6127, another high- $B$ RPP (Gonzalez et al. 2005; Ng et al. 2012, in preparation). It is even hotter than the magnetar XTE J1810-197, although its temperature is well below those of the other magnetars. In particular, however, it is far hotter than multiple other RPPs of comparable age but smaller magnetic field. Indeed, as noted by Zhu et al. (2011), high- $B$ RPPs in general show a trend toward higher blackbody temperatures when compared with lower- $B$ RPPs of similar age. This suggests that the magnetic field affects the observed thermal properties of RPPs, as is expected if the star is actively heated by field decay. However, enhanced thermal emission could also be a result of passive effects such as magnetically altered thermal conductivity (Geppert et al. 2006; Page et al. 2007) without any active field decay. Distinguishing between active and passive explanations for the enhanced thermal emission in high- $B$ RPPs could be achieved via detailed modelling, or perhaps by observing other signatures of active field decay, such as magnetar-like bursts in high-B RPPs. Indeed this has been observed in one source already (Gavriil et al. 2008). 


\subsection{PSRs B1845-19 and J1001-5939}

We have reported on X-ray observations of two rotation-powered pulsars, PSRs B1845-19 and J1001-5939, taken with the XMM-Newton and Chandra telescopes, respectively. In both cases we failed to detect the pulsars and set upper limits on their $k T$, assuming a blackbody spectrum. These two sources were selected for observation because their spin-derived properties are very similar to those of the XINSs, but as shown in Figure 5, this is not the case in the X-ray band. The $k T$ upper limits we find for our two pulsars imply that they are significantly cooler than the bulk of the XINS population, although we cannot exclude the possibility that their temperatures are similar to that of the coolest known XINS, RX J0420.0-5022 (Haberl et al. 2004).

In the magneto-thermal evolution model of Pons et al. (2009) and Popov et al. (2010), the XINSs are born with magnetic fields much higher than what we currently measure $\left(\gtrsim 10^{14} \mathrm{G}\right.$, possibly including a significant toroidal component). As they age, the field decays and heats the surface of the star. A reasonable explanation, then, for PSRs B1845-19 and J1001-5939 to have much lower temperatures than most of the XINSs, is that they have similar ages but were born with lower magnetic fields. They would therefore experience less heating from magnetic-field decay and their thermal evolution would more closely resemble the standard cooling curves of non-magnetic neutron stars. This explanation was also suggested by Kaplan \& van Kerkwijk (2011) to account for the low temperature of RX J0420.0-5022, and indeed the upper limits on our sources do allow for the possibility that they and RX J0420.0-5022 followed similar evolutionary tracks.

Another possible explanation is that PSRs B1845-19 and J1001-5939 do not have similar ages to the XINSs. The above models assume that the XINSs have true ages of $\sim 0.5-1 \mathrm{Myr}$, based on kinematic age estimates for some of them (Tetzlaff et al. 2011). These kinematic ages are smaller than those inferred from spin-down (1.5-4 Myr). If the kinematic ages are correct, then it is possible that our radio pulsar sources have true ages considerably closer to their characteristic ages (possibly as a result of different evolution as above). In that case, they have lower temperatures than most or all of the XINSs simply because they are older and have cooled further. For a related scenario see Turolla et al. (2011), who, based on magneto-thermal evolution, model the low-field magnetar SGR 0418+5729 (Rea et al. 2010) as being 1-1.5 Myr old and having undergone substantial magneticfield decay.

\section{Conclusion}

In summary, we have presented results from XMM-Newton and Chandra observations of three high- $B$ radio pulsars: PSRs J1734-3333, B1845-19, and J1001-5939. Our observation of PSR J1734-3333 was taken to follow up on its detection in 2009. Although we do not detect Xray pulsations from the source, we find that it has a thermal spectrum with $k T=300 \pm 60 \mathrm{eV}$, significantly higher than predicted by cooling models. Atmosphere models (nsa and nsmax) yield lower temperatures that are consistent with standard cooling, but the nsa model in particular 
predicts too high of a distance or column density. Comparing PSR J1734-3333 with other RPPs, we find it has a significantly higher blackbody temperature than nearly every other radio pulsar for which such a measurement has been made, particularly other RPPs of similar age but lower magnetic field. This result suggests that a high magnetic field can affect the observed thermal properties of pulsars, for example by heating of the surface due to magnetic field decay, or by passive atmospheric effects. PSR B1845-19 and PSR J1001-5939 were not detected in our X-ray observations. Assuming blackbody emission from a 10-km radius, we derive $3 \sigma$ upper limits on their temperatures of 48 and $56 \mathrm{eV}$, consistent with standard cooling curves. The two radio pulsars have similar spin-derived properties to the XINSs, but the temperature limits we find are significantly below the observed temperatures of all the XINSs except RX J0420.0-5022. We conclude that these radio pulsars may have been born with lower magnetic fields than any of the XINSs (with the possible exception of RX J0420.0-5022), experienced less magnetic-field-decay heating, and therefore their evolution more closely resembled that of ordinary radio pulsars.

Part of this work was performed under the auspices of the U.S. Department of Energy by Lawrence Livermore National Laboratory under Contract No. DE-AC52-07NA27344. VMK holds the Lorne Trottier Chair in Astrophysics and Cosmology, and a Canada Research Chair, a Killam Research Fellowship, and acknowledges additional support from an NSERC Discovery Grant, from FQRNT via le Centre de Recherche Astrophysique du Québec and the Canadian Institute for Advanced Research. Support for this work was provided by NASA through Chandra award GO1$12083 X$.

Facilities: $C X O$ (ACIS), XMM (EPIC)

\section{REFERENCES}

Cordes, J. M., \& Lazio, T. J. W. 2002, arXiv:astro-ph/0207156

Drimmel, R., Cabrera-Lavers, A., López-Corredoira, M. 2003, A\&A, 409, 205

Espinoza, C. M., Lyne, A. G., Kramer, M. et al. 2011, ApJ, 741, L13

Garmire, G. P., Bautz, M. W., Ford, P. G., et al. 2003, Proc. SPIE, 4851, 28

Gavriil, F. P., Gonzalez, M. E., Gotthelf, E. V., et al. 2008, Science, 319, 1802

Geppert, U., Küker, M., \& Page, D. 2006, A\&A, 457, 937

Gonzalez, M. E., Kaspi, V. M., Camilo, F., et al. 2005, ApJ, 630, 489

Harding, A. K., \& Muslimov, A. G. 2001, ApJ, 556, 987

Haberl, F. 2007, Ap\&SS, 308, 181 
Haberl, F., Motch, C., Zavlin, K., et al. 2004, A\&A, 424, 635

Ho, W. C. G., Potekhin, A. Y., \& Chabrier, G. 2008, ApJS, 178, 102

Jansen, F., et al. 2001, A\&A, 365, L1

Kaplan, D. L., \& van Kerkwijk, M. H. 2009, ApJ, 705, 798

Kaplan, D. L., \& van Kerkwijk, M. H. 2011, ApJ, 740, L30

Kaspi, V. M. 2010, PNAS, 107, 7147

Kaspi, V. M., Chakrabarty, D., \& Steinberger, J. 1999, ApJ, 525, L33

Levin, L., Bailes, M., Bates, S., et al. 2010, ApJ, 721, L33

Livingstone, M. A., Scholz, P., Kaspi, V. M., et al. 2011, ApJ, 743, L38

Lorimer, D. R., Faulkner, A. J., Lyne, A. G., et al. 2006, MNRAS, 372, 777

Manchester, R. N., Lyne, A. G., Taylor, J. H., et al. 1978, MNRAS, 185, 409

Manchester, R. N., Lyne, A. G., Camilo, F., et al. 2001, MNRAS, 328, 17

Mereghetti, S. 2008, A\&A Rev., 15, 225

Morris, D. J., Hobbs, G., Lyne, A. G., et al. 2002, MNRAS, 335, 275

Ng, C.-Y., Kaspi, V. M., Ho, W. C. G., et al. 2012, ApJ, in press

Olausen, S. A., Kaspi, V. M., Lyne, A. G., \& Kramer, M. 2010, ApJ, 725, 985

Page, D., Geppert, U., \& Manfred, K. 2007, Ap\&SS, 308, 403

Page, D., Geppert, U., \& Weber, F. 2006, Nucl. Phys. A, 777, 497

Pavlov, G. G., Shibanov, Y. A., Zavlin, V. E., \& Meyer, R. D. 1995, in The Lives of the Neutron Stars, ed. M. A. Alpar, Ü. Kiziloğlu, \& J. van Paradijs (NATO ASI Ser. C, Vol. 450; Dordrecht: Kluwer), 71

Pons, J. A., Miralles, J. A., \& Geppert, U. 2009, A\&A, 496, 207

Popov, S. B., Pons, J. A., Miralles, J. A., et al. 2010, MNRAS, 401, 2675

Predehl, P., \& Schmitt, J. H. M. M. 1995, A\&A, 293, 889

Rea, N., Esposito, P., Turolla, R., et al. 2010, Science, 330, 944

Rea, N., Israel, G. L., Esposito, P., et al. 2012, ApJ, 754, 27 
Scholz, P., Ng, C.-Y., Livingstone, M. A., et al. 2012, ApJ, in press

Strüder, L., et al. 2001, A\&A, 365, L18

Speagle, J. S., Kaplan, D. L., \& van Kerkwijk, M. H. 2011, ApJ, 743, 183

Tetzlaff, N., Eisenbeiss, T., Neuhäuser, R., \& Hohle, M. M. 2011, MNRAS, 417, 617

Thompson, C., \& Duncan, R. C. 1995, MNRAS, 275, 255

Thompson, C., \& Duncan, R. C. 1996, ApJ, 473, 322

Thompson, C., Lyutikov, M., \& Kulkarni, S. R. 2002, ApJ, 574, 332

Turner, M. J. L., et al. 2001, A\&A, L27

Turolla, R. 2009, in Neutron Stars and Pulsars, ed. W. Becker (Astrophysics and Space Science Library, Vol. 357; Berlin: Springer), 141

Turolla, R., Zane, S., Pons, J. A., et al. 2011, ApJ, 740, 105

van Kerkwijk, M. H., \& Kaplan, D. L. 2008, ApJ, 673, L163

Woods, P. M., \& Thompson, C. 2006, in Compact Stellar X-ray Sources, ed. W. H. G. Lewin \& M. van der Klis (Cambridge: Cambridge Univ. Press), 547

Zhu, W. W., Kaspi, V. M., McLaughlin, M. A., et al. 2011, ApJ, 734, 44

This preprint was prepared with the AAS IATEX macros v5.2. 
Table 1. Summary of X-ray Observations

\begin{tabular}{|c|c|c|c|c|c|c|}
\hline Target & Telescope & Obs ID & Date & Detector & Time Resolution (s) & Exposure $(\mathrm{ks})^{\mathrm{a}}$ \\
\hline \multirow[t]{4}{*}{ J1734-3333 } & $X M M$ & 0553850101 & 2009 Mar 9 & pn & 0.048 & 8.7 \\
\hline & $X M M$ & 0653320101 & 2011 Mar 11 & pn & 0.048 & 42.8 \\
\hline & & & & MOS1 & 2.6 & 59.8 \\
\hline & & & & MOS1 & 2.6 & 64.5 \\
\hline \multirow[t]{3}{*}{ B1845-19 } & $X M M$ & 0653300101 & 2011 Mar 16 & pn & 0.048 & 28.2 \\
\hline & & & & MOS1 & 2.6 & 27.3 \\
\hline & & & & MOS2 & 2.6 & 27.3 \\
\hline J1001-5939 & Chandra & 12561 & 2011 Oct 8 & ACIS-S & 0.44 & 17.2 \\
\hline
\end{tabular}

aThe exposure time is dead-time corrected and has intervals of high background flaring removed.

Table 2. $\quad$ Spectral Models for PSR J1734-3333

\begin{tabular}{cccc}
\hline \hline Parameter & Blackbody & $\mathrm{nsa}^{\mathrm{a}}$ & $\mathrm{nsmax}^{\mathrm{b}}$ \\
\hline$N_{H}\left(10^{22} \mathrm{~cm}^{-2}\right)$ & $0.67_{-0.25}^{+0.35}$ & $0.83_{-0.29}^{+0.41}$ & $0.88_{-0.28}^{+0.40}$ \\
$k T^{\infty}(\mathrm{keV})$ & $0.30 \pm 0.06$ & $0.14 \pm 0.05$ & $0.13_{-0.04}^{+0.05}$ \\
$R^{\infty}(\mathrm{km})$ & $0.45_{-0.20}^{+0.55}$ & $13($ fixed $)$ & $3.2_{-2.0}^{+8.9}$ \\
Distance $\left.\mathrm{ckpc}^{\mathrm{c}}\right)$ & $\cdots$ & $27_{-20}^{+50}$ & $\cdots$ \\
$\chi^{2}(\mathrm{dof})$ & $34.7(44)$ & $34.6(44)$ & $34.3(44)$ \\
$f_{\text {abs }}\left(10^{-15} \mathrm{erg} \mathrm{s}^{-1} \mathrm{~cm}^{-2}\right)^{\mathrm{d}}$ & $9.9 \pm 1.2$ & $9.9_{-1.2}^{+1.1}$ & $9.8_{-1.1}^{+1.2}$ \\
$f_{\text {unabs }}\left(10^{-14} \mathrm{erg} \mathrm{s}^{-1} \mathrm{~cm}^{-2}\right)^{\mathrm{d}}$ & $3.6_{-1.4}^{+3.7}$ & $5.6_{-2.7}^{+9.9}$ & $6.6_{-3.4}^{+11.6}$ \\
\hline
\end{tabular}

The nsa model for a pulsar with $B=10^{13} \mathrm{G}$.

${ }^{\text {b}}$ The nsmax model for a pulsar with $B=2 \times 10^{13} \mathrm{G}$.

${ }^{\mathrm{c}}$ The distance to the pulsar is fit for in the nsa model, while in the other two models the DM distance of $6.1 \mathrm{kpc}$ is used to determine the radius of emission.

${ }^{\mathrm{d}}$ Absorbed and unabsorbed fluxes are given in the $0.5-2.0 \mathrm{keV}$ band. 


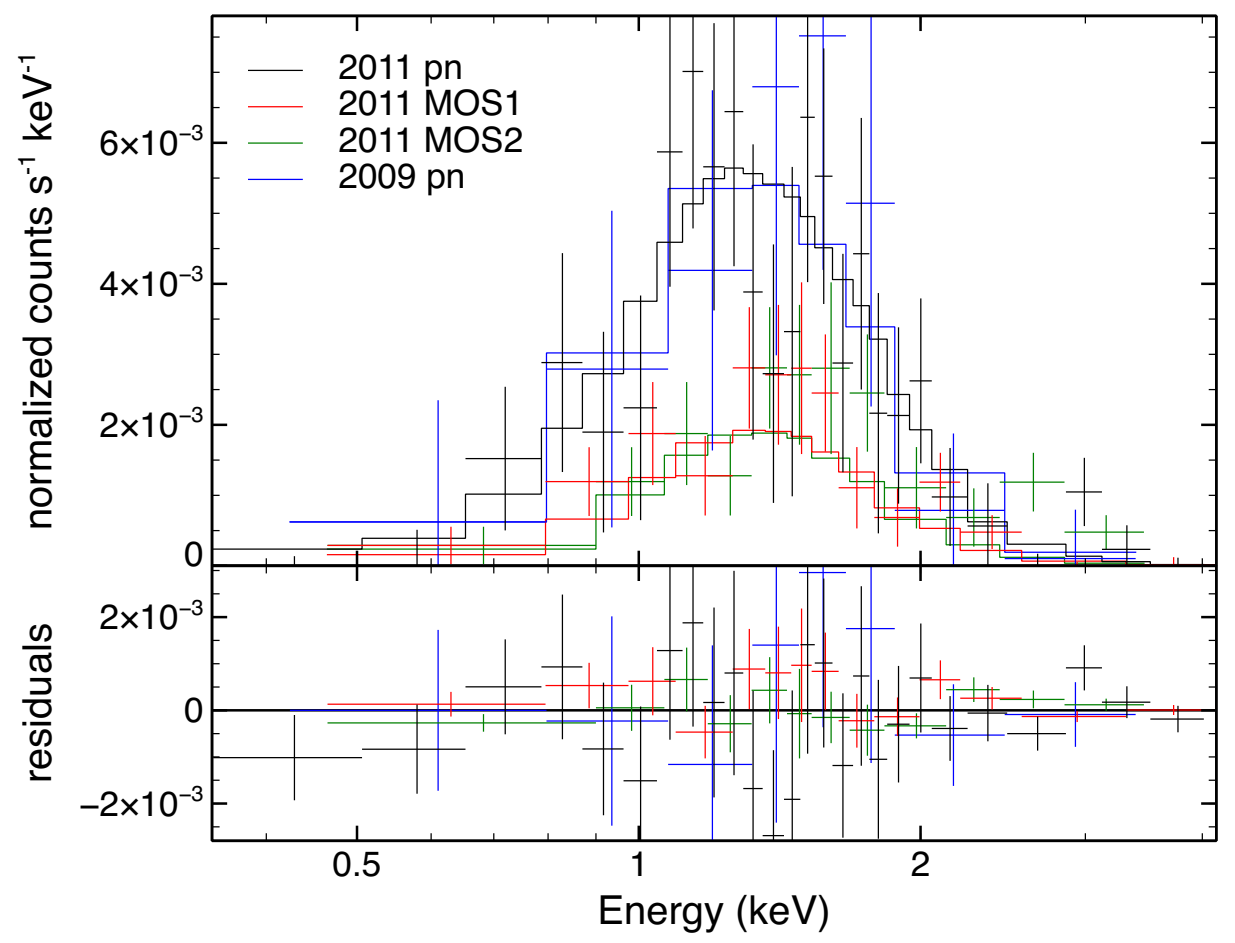

Fig. 1. - XMM spectrum of PSR J1734-3333 with the best-fit blackbody model. The bottom panel shows the residuals in units of counts s $\mathrm{seV}^{-1}$. Error bars are at the $1 \sigma$ confidence level. 


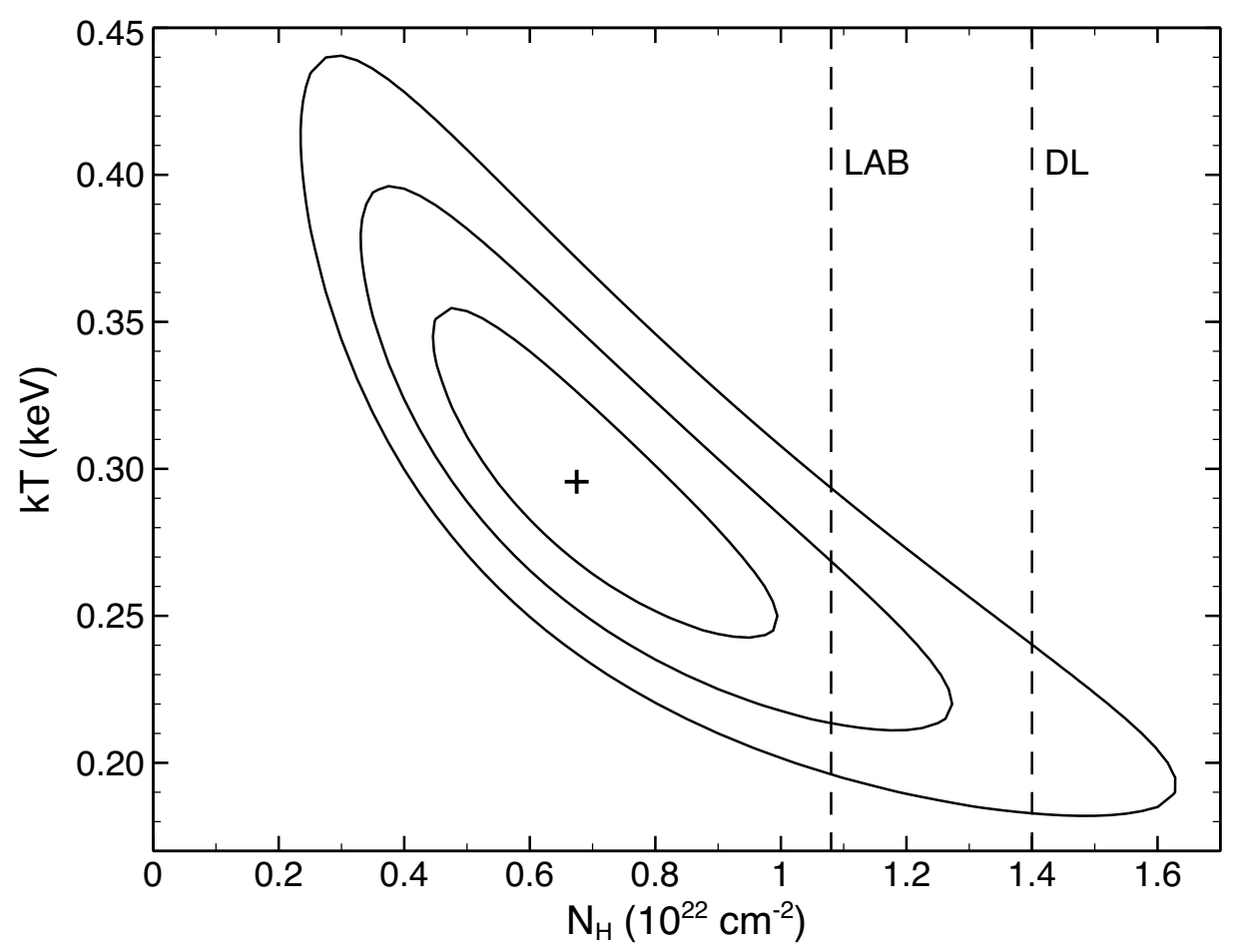

Fig. 2. $-1 \sigma, 2 \sigma$, and $3 \sigma$ confidence contours for $k T$ and $N_{\mathrm{H}}$ from fitting an absorbed blackbody model to the X-ray spectrum of PSR J1734-3333. The cross marks the best-fit value, and the dashed lines mark the total $N_{\mathrm{H}}$ along the line-of-sight to the pulsar according to the Leiden/Argentine/Bonn (labeled LAB) and Dickey \& Lockman (labeled DL) Galactic H I surveys.
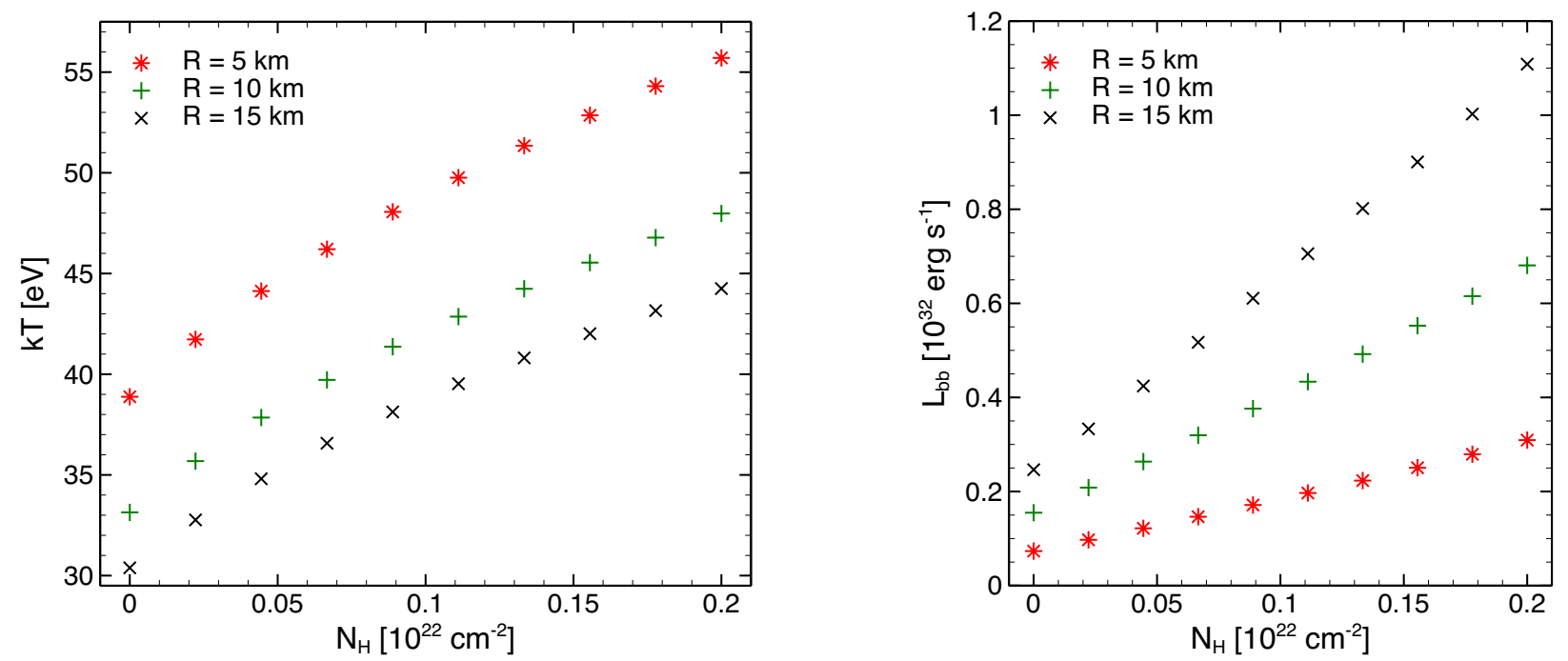

Fig. 3. - $3 \sigma$ upper limits on the blackbody temperature $k T$ (left) and bolometric luminosity (right) for PSR B1845-19. 

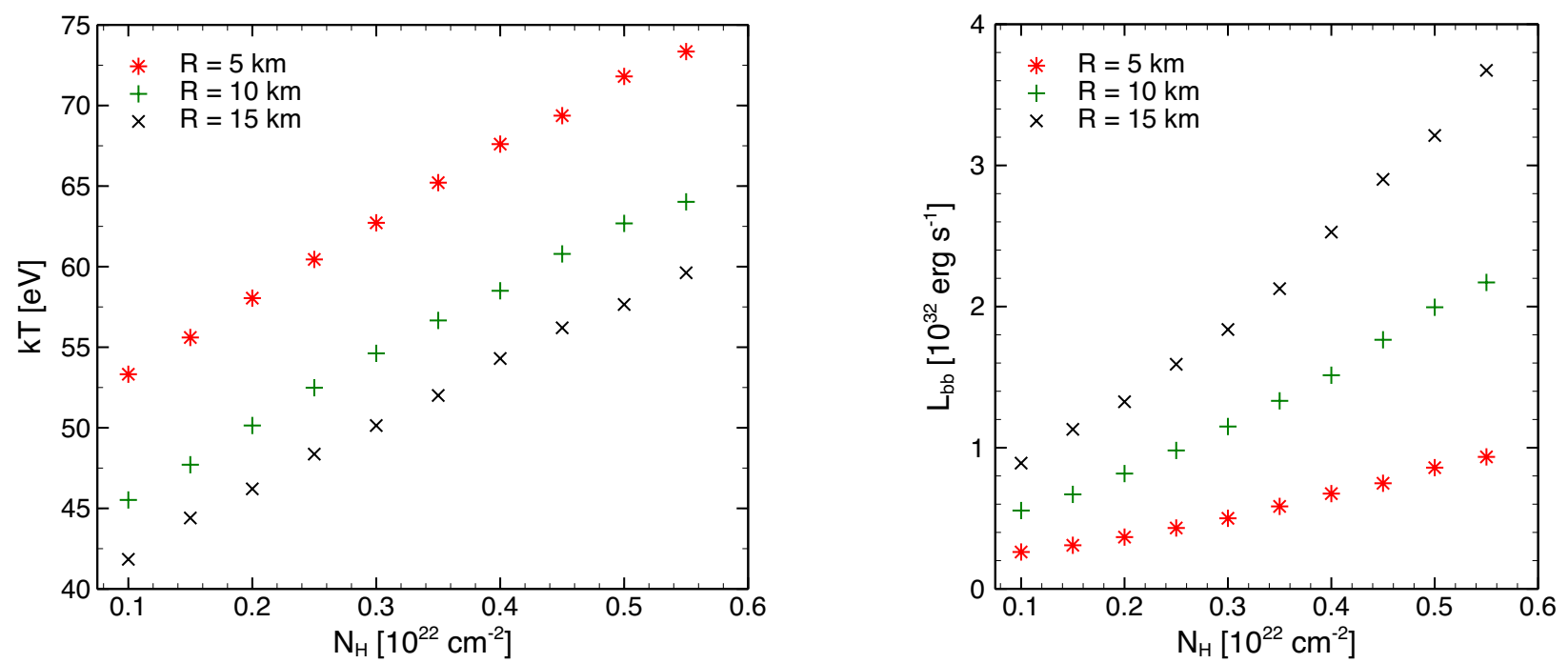

Fig. 4. - $3 \sigma$ upper limits on the blackbody temperature $k T$ (left) and bolometric luminosity (right) for PSR J1001-5939. 


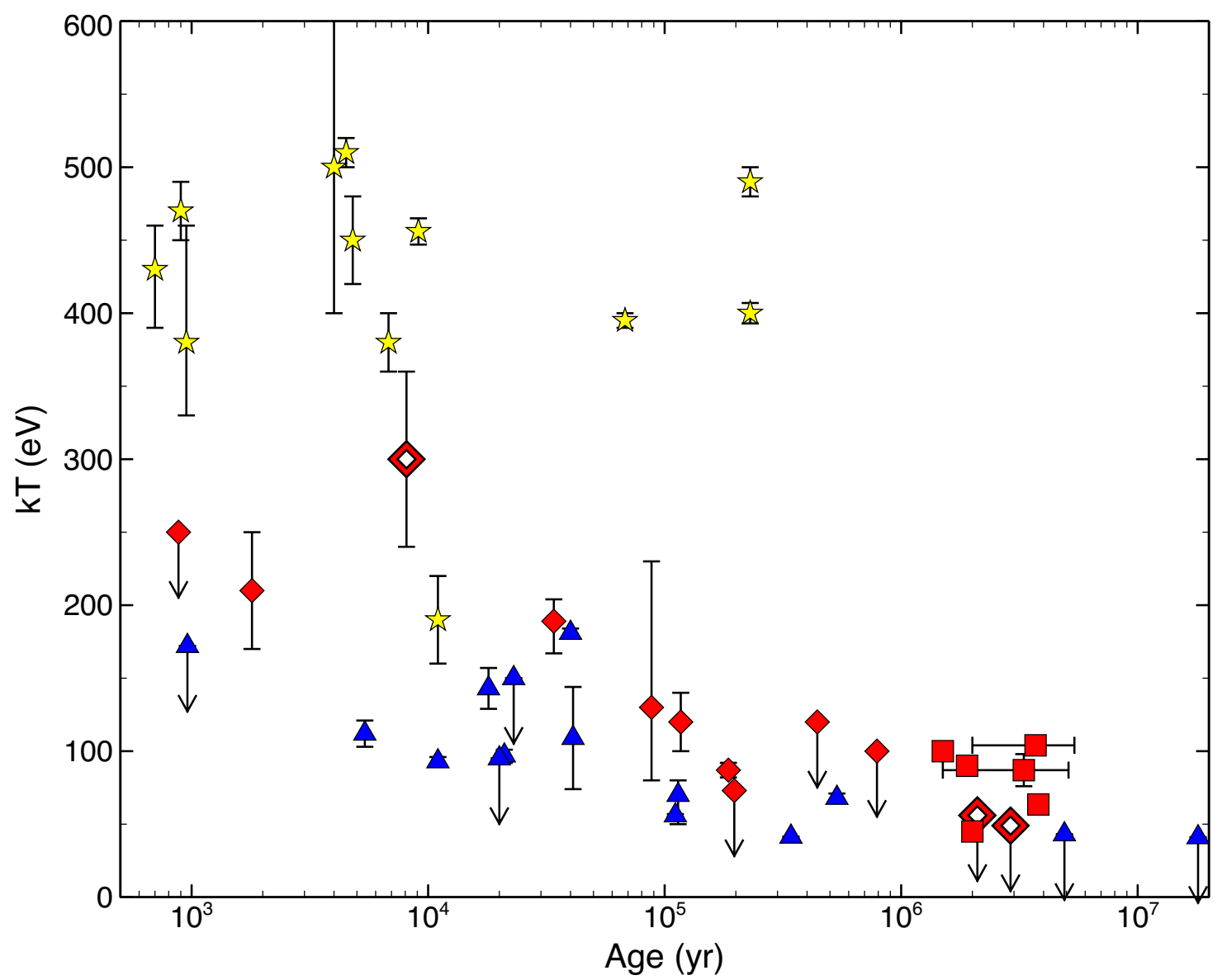

Fig. 5.- Blackbody temperatures vs. characteristic ages for normal pulsars (blue triangles), high- $B$ pulsars ( $B \geq 10^{13} \mathrm{G}$; red diamonds), XINSs (red squares), and magnetars (yellow stars). Magnetars were plotted only if they had a measurement of $k T$ in quiescence with uncertainties of $0.1 \mathrm{keV}$ or less (1E 1048.1-5937, 1E 1547.0-5408, 1E 1841-045, 1E 2259+586 1RXS J170849.0-400910, 4U 0142+61, CXOU J010043.1-721134, CXOU J164710.2-455216, CXOU J171405.7-381031, PSR 1622-4950, SGR 1900+14, and XTE J1810-197), based on data taken from http://www.physics.mcgill.ca/ pulsar/magnetar/main.html. The data for the other sources are taken from Zhu et al. (2011) with the addition of PSR J0726-2612 (Speagle et al. 2011), an updated temperature for PSR J1119-6127 (Ng et al. 2012), an updated timing solution for RX J0420.0-5022 (Kaplan \& van Kerkwijk 2011), and data from this work. The three high-B pulsars described in this paper are represented by large red diamonds with holes. 